Pacific Journal of Mathematics

ON THE EVALUATION OF PERMANENTS 


\section{ON THE EVALUATION OF PERMANENTS}

\section{NAtália Bebiano*}

Two identities involving permanents are obtained. One of them is used to deduce in a simple way, two well known formulas for the evaluation of permanents, namely the formulas of Binet and Minc and of Ryser.

1. Notation. Let $A=\left[a_{i j}\right]$ be an $n \times n$ matrix. The permanent of $A$ is defined by

$$
\operatorname{per}(A)=\sum_{\sigma \in S_{n}} \prod_{i=1}^{n} a_{i \sigma(i)},
$$

where $S_{n}$ is the symmetric group of degree $n$.

Let $\Gamma_{r, n}$ denote the set of all $n^{r}$ sequences $\omega=\left(\omega_{1}, \cdots, \omega_{r}\right)$ of integers satisfying $1 \leqq \omega_{i} \leqq n$. Let $Q_{r, n}$ be the set of those sequences in $\Gamma_{r, n}$ which are strictly increasing.

By $G(n)$ we denote the set of all nondecreasing sequences of positive integers $\left(t_{1}, \cdots, t_{k}\right)$ such that $t_{1}+\cdots+t_{k}=n$.

Given an $n \times n$ matrix $A$ and nonnegative integers $\alpha_{1}, \cdots, \alpha_{n}$ $\left(\beta_{1}, \cdots, \beta_{n}\right)$ satisfying $\alpha_{1}+\cdots+\alpha_{n}=n\left(\beta_{1}+\cdots+\beta_{n}=n\right)$, we represent by $A\left(\alpha_{1}, \cdots, \alpha_{n}\right)\left(A\left(\beta_{1}, \cdots, \beta_{n}\right)\right)$ the matrix obtained from $A$ by repeating its first row (column) $\alpha_{1}\left(\beta_{1}\right)$ times, its second row (column) $\alpha_{2}\left(\beta_{2}\right)$ times $\cdots$ and its $n$th row (column) $\alpha_{n}\left(\beta_{n}\right)$ times. If $\alpha_{i}=0\left(\beta_{i}=0\right)$ the $i$ th row (column) of $A$ is omitted.

Given an $n \times n$ matrix $A$ and nonnegative integers $\alpha_{1}, \cdots, \alpha_{n}$, $\beta_{1}, \cdots, \beta_{n} \quad$ satisfying $\alpha_{1}+\cdots+\alpha_{n}=n, \quad \beta_{1}+\cdots+\beta_{n}=n$, let $A\left(\alpha_{1}, \cdots, \alpha_{n} ; \beta_{1}, \cdots, \beta_{n}\right)$ denote the $n \times n$ matrix obtained from $A$ by repeating its first row $\alpha_{1}$ times, $\cdots$, its $n$th row $\alpha_{n}$ times and also its first column $\beta_{1}$ times $\cdots$ its $n$th column $\beta_{n}$ times. Again $\alpha_{i}=0$ or $\beta_{i}=0$ means that the $i$ th row or the $i$ th column of $A$ is omitted.

Given the integers $\alpha_{i}, 1 \leqq i \leqq n$, such that $\sum_{i=1}^{n} \alpha_{i}=n$, let $R_{\alpha_{1}, \cdots \alpha_{n}}=\left(j_{1}, \cdots, j_{n}\right)$ represent the nondecreasing sequence of nonnegative integers $\left(j_{1}, \cdots, j_{n}\right)$ where $i$ occurs with multiplicity $\alpha_{i}$.

2. Two identities involving permanents. Let $A$ be an $n \times n$ matrix and

$$
Z=\prod_{i=1}^{n} Z_{i}
$$

with

* The author is a member of the Centro de Matemática da Universidade de Coimbra 


$$
Z_{i}=\sum_{j=1}^{n} a_{i j} x_{j}, \quad i=1, \cdots, n .
$$

Since $Z$ is an homogeneous polynomial of degree $n$ in $x_{1}, \cdots, x_{n}$ we can write

$$
Z=\sum_{\beta_{1}, \ldots, \beta_{n}} x_{\beta_{1}, \cdots, \beta_{n}} \frac{x_{1}^{\beta_{1}}}{\beta_{1} !} \frac{x_{2}^{\beta_{2}}}{\beta_{2} !} \cdots \frac{x_{n}^{\beta_{n}}}{\beta_{n} !},
$$

where the summation is over all sequences $\left(\beta_{1}, \cdots, \beta_{n}\right)$ of nonnegative integers satisfying $\beta_{1}+\cdots+\beta_{n}=n$. The coefficient $x_{\beta_{1}, \cdots, \beta_{n}}$ is equal to

$$
\frac{\partial^{n} Z}{\partial x_{j_{1}} \partial x_{j_{2}} \cdots \partial x_{j_{n}}}, \quad\left(j_{1}, \cdots, j_{n}\right)=R_{\beta_{1}, \cdots, \beta_{n}}
$$

as can be seen from the following

$$
\begin{aligned}
\frac{\partial^{n} Z}{\partial x_{j_{1}} \partial x_{j_{2}} \cdots \partial x_{j_{n}}} & =\frac{\partial^{\beta_{1}+\cdots+\beta_{n}} Z}{\partial x_{1}^{\beta_{1}} \cdots \partial x_{n}^{\beta_{n}}} \\
& =x_{\beta_{1}, \cdots, \beta_{n}}\left(\frac{1}{\beta_{1} !} \frac{\partial^{\beta_{1}} x_{1}^{\beta_{1}}}{\partial x_{1}^{\beta_{1}}}\right) \cdots\left(\frac{1}{\beta_{n} !} \frac{\partial^{\beta_{n}} x_{n}^{\beta_{n}}}{\partial x_{n}^{\beta_{n}}}\right) \\
& =x_{\beta_{1}, \cdots, \beta_{n}} \frac{\beta_{1} !}{\beta_{1} !} \cdots \frac{\beta_{n} !}{\beta_{n} !} \\
& =x_{\beta_{1}, \cdots, \beta_{n}} .
\end{aligned}
$$

We now compute $\partial^{n} Z / \partial x_{j_{1}} \cdots \partial x_{j_{n}},\left(j_{1}, \cdots, j_{n}\right)=R_{\beta_{1}, \ldots, \beta_{n}}$. We begin by assuming $\left(j_{1}, \cdots, j_{n}\right)=R_{1,1, \cdots, 1}$. Since $Z_{i}$ is a linear function of the $x_{j}$ 's we have

$$
\frac{\partial^{2} Z_{i}}{\partial x_{k} \partial x_{h}}=0
$$

for any $k$ and $h$ and by differentiation

$$
\begin{aligned}
\frac{\partial^{n} Z}{\partial x_{j_{1}} \partial x_{j_{2}} \cdots \partial x_{j_{n}}} & =\frac{\partial^{n} Z}{\partial x_{1} \partial x_{2} \cdots \partial x_{n}} \\
& =\sum_{\sigma} \frac{\partial Z_{\sigma(1)}}{\partial x_{1}} \frac{\partial Z_{\sigma(2)}}{\partial x_{2}} \cdots \frac{\partial Z_{\sigma(n)}}{\partial x_{n}},
\end{aligned}
$$

where $\sigma \in S_{n}$. But

$$
\frac{\partial Z_{o(i)}}{\partial x_{i}}=a_{\sigma(i) i}
$$

and therefore

$$
\frac{\partial^{n} Z}{\partial x_{1} \cdots \partial x_{n}}=\sum_{\sigma \in S_{n}} \prod_{i=1}^{n} a_{o(i) i}=\operatorname{per}(A) .
$$


Let now $\left(j_{1}, \cdots, j_{n}\right)=R_{\beta_{1}, \cdots, \beta_{n}}$. Again by differentiation and recalling (2.2) we have

$$
\begin{aligned}
\frac{\partial^{n} Z}{\partial x_{j_{1}} \partial x_{j_{2}} \cdots \partial x_{j_{n}}} & =\sum_{\sigma \in S_{n}} \prod_{i=1}^{n} \frac{\partial Z_{\sigma(i)}}{\partial x_{j_{i}}} \\
& =\sum_{\sigma \in S_{n}} \prod_{i=1}^{n} a_{\sigma(i) j_{i}} \\
& =\operatorname{per}\left(A\left(\beta_{1}, \cdots, \beta_{n}\right)\right) .
\end{aligned}
$$

From (2.1), (2.3) and (2.4) we obtain the following identity

$$
\prod_{i=1}^{n}\left(\sum_{j=1}^{n} a_{i j} x_{j}\right)=\sum_{\beta_{1}, \ldots, \beta_{n}} \operatorname{per}\left(A\left(\beta_{1}, \cdots, \beta_{n}\right)\right) \frac{x_{1}^{\beta_{1}}}{\beta_{1} !} \cdots \frac{x_{n}^{\beta_{n}}}{\beta_{n} !},
$$

where the summation is over all the sequences $\left(\beta_{1}, \cdots, \beta_{n}\right)$ of nonnegative integers satisfying $\sum_{i=1}^{n} \beta_{i}=n$.

Now we shall deduce from (2.5), the following identity expressing the $n$th power of a bilinear form as a polynomial with permanents as coefficients,

$$
\begin{aligned}
\frac{1}{n !}\left(\sum_{i, j=1}^{n} x_{i} a_{i j} y_{j}\right)^{n}= & \sum_{\alpha, \beta} \operatorname{per}\left(A\left(\alpha_{1}, \cdots, \alpha_{n} ; \beta_{1}, \cdots, \beta_{n}\right)\right) \frac{x_{1}^{\alpha_{1}}}{\alpha_{1} !} \\
& \times \cdots \frac{x_{n}^{\alpha_{n}}}{\alpha_{n} !} \frac{y_{1}^{\beta_{1}}}{\beta_{1} !} \cdots \frac{y_{n}^{\beta_{n}}}{\beta_{n} !}
\end{aligned}
$$

where $\alpha=\left(\alpha_{1}, \cdots, \alpha_{n}\right), \beta=\left(\beta_{1}, \cdots, \beta_{n}\right)$, with $\alpha_{i}, \beta_{i} \geqq 0 i=1, \cdots, n$ and $\sum_{i=1}^{n} \alpha_{i}=\sum_{i=1}^{n} \beta_{i}=n$.

Indeed, by the multinomial expansion we have

$$
\frac{1}{n !}\left(\sum_{i, j=1}^{n} x_{i} a_{i j} y_{j}\right)^{n}=\sum_{\alpha} \prod_{i=1}^{n}\left[\frac{x_{i}^{\alpha_{i}}}{\alpha_{i} !}\left(\sum_{j=1}^{n} a_{i j} y_{j}\right)^{\alpha_{i}}\right],
$$

where $\alpha_{i} \geqq 0, i=1, \cdots, n$ and $\sum_{i=1}^{n} \alpha_{i}=n$. Let $\left(h_{1}, \cdots, h_{n}\right)=R_{\alpha_{1}, \cdots, \alpha_{n}}$. We have

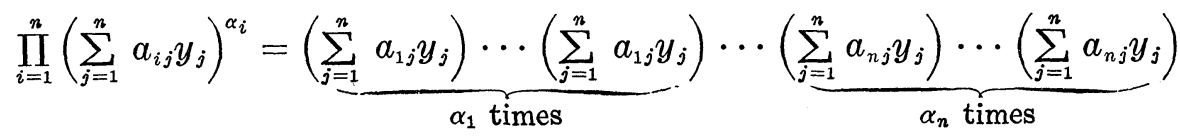

$$
\begin{aligned}
& =\left(\sum_{j=1}^{n} a_{h_{1} j} y_{j}\right) \cdots\left(\sum_{j=1}^{n} a_{h_{n} j} y_{j}\right) \\
& =\prod_{i=1}^{n}\left(\sum_{j=1}^{n} a_{h_{i} j} y_{j}\right) \text {. }
\end{aligned}
$$

Substituting (2.8) into (2.7), applying the identity (2.5) to $\prod_{i=1}^{n}\left(\sum_{j=1}^{n} a_{h_{i} j} y_{j}\right)$ and noting that the matrix $A=\left(a_{i j}\right)$ has been replaced by the matrix $A\left(\alpha_{1}, \cdots, \alpha_{n}\right)=\left(a_{h_{i} j}\right)$ we obtain 


$$
\begin{aligned}
\frac{1}{n !}\left(\sum_{i, j=1}^{n} x_{i} \alpha_{i j} y_{j}\right)^{n}=\sum_{\alpha} \prod_{i=1}^{n} \frac{x_{i}^{\alpha_{i}}}{\alpha_{i} !} \prod_{i=1}^{n}\left(\sum_{j=1}^{n} \alpha_{h_{i} j} y_{j}\right) \\
=\sum_{\alpha} \prod_{i=1}^{n} \frac{x_{i}^{\alpha_{i}}}{\alpha_{i} !} \sum_{\beta} \operatorname{per}\left(A\left(\alpha_{1}, \cdots, \alpha_{n} ; \beta_{1}, \cdots, \beta_{n}\right)\right) \frac{y_{1}^{\beta_{1}}}{\beta_{1} !} \cdots \frac{y_{n}^{\beta_{n}}}{\beta_{n} !} .
\end{aligned}
$$

This proves (2.6).

3. About the Binet-Minc formula. As we have seen per $A=\partial^{n} Z / \partial x_{1} \cdots \partial x_{n}$ which is equivalent to per $A=\operatorname{coef}_{x_{1} \cdots x_{n}} \prod_{i=1}^{n} \times$ $\left(\sum_{j=1}^{n} a_{i j} x_{j}\right)$. This result for the case $n=3$, is due to Muir. Let us evaluate $\partial^{n} Z / \partial x_{1} \cdots \partial x_{n}$. Let again $Z=\prod_{i=1}^{n} Z_{i}$ and $Z_{i}=\sum_{j=1}^{n} a_{i j} x_{j}$, $i=1, \cdots, n$. Let $T_{1}, \cdots, T_{k}$ be a partition of $\{1, \cdots, n\}$ such that

$$
T_{j}=\left\{w_{1}^{(j)}, \cdots, w_{s}^{(j)}\right\} \in Q_{s, n},
$$

where $s=t_{j}, j=1, \cdots, k$ and $t_{1} \leqq \cdots \leqq t_{k}$.

We define

$$
\phi^{\left(w_{1}^{(j)}\right)}=\sum_{h=1}^{n} \frac{1}{Z_{h}} \frac{\partial Z_{h}}{\partial x_{w_{1}^{(j)}}^{(j)}}
$$

and

$$
\phi^{\left(T_{j}\right)}=\frac{\partial^{t_{j}-1} \dot{\phi}^{(j)}}{\partial x_{w_{2}^{(j)}} \cdots \partial x_{w_{s}^{(j)}}}
$$

with $s=t_{j}, j=1, \cdots, k$.

It is clear that

$$
\phi^{\left(w_{1}^{(j)}\right)}=\sum_{h=1}^{n} \frac{a_{h w_{1}^{(j)}}}{Z_{h}} .
$$

On the other hand, it is easy to show (by induction) that

$$
\phi^{\left(T_{j}\right)}=(-1)^{t_{j}-1}\left(t_{j}-1\right) ! \sum_{h=1}^{n} \frac{a_{h w_{1}^{(j)}} \cdots a_{h w_{s}^{(j)}}}{Z_{h}^{t_{j}}}
$$

$s=t_{j}, j=1, \cdots, k$. In fact, suppose

$$
\phi^{\left(T_{j}-w_{s}^{(j)}\right)}=\frac{\partial^{s-2} \phi^{\left(w_{1}^{(j)}\right)}}{\partial x_{\omega_{2}^{(j)}} \cdots \partial x_{w_{s-1}^{(j)}}}=(-1)^{s-2}(s-2) ! \sum_{h=1}^{n} \frac{a_{h w_{1}^{(j)}} \cdots a_{h w_{s-1}^{(j)}}}{Z_{h}^{s-1}},
$$

where $s=t_{j}$, and $T_{j}-w_{s}^{(j)}$ denotes the set $\left\{w_{1}^{(j)}, \cdots, w_{s-1}^{(j)}\right\} . \quad$ By differentiating (3.6) with respect to $x_{w_{s}^{(j)}}$ we obtain

$$
\begin{aligned}
\frac{\partial^{s-1} \phi^{\left(w_{1}^{(j)}\right)}}{\partial x_{w_{2}^{(j)}} \cdots \partial x_{w_{s-1}^{(j)}} \partial x_{w_{s}^{(j)}}} & =(-1)^{s-1}(s-1) ! \sum_{h=1}^{n} \frac{a_{h w_{1}^{(j)} \cdots a_{h w_{s-1}^{(j)}}} \frac{\partial Z_{h}}{Z_{h}^{s}}}{x_{w_{s}^{(j)}}} \\
& =(-1)^{s-1}(s-1) ! \sum_{h=1}^{n} \frac{a_{h w_{1}^{(j)} \cdots a_{h w_{s-1}^{(j)}}} a_{h w_{s}^{(j)}}}{Z_{h}^{s}}
\end{aligned}
$$


and (3.5) holds.

We finally prove (by induction) that

$$
\frac{\partial^{n} Z}{\partial x_{1} \cdots \partial x_{n}}=Z \sum_{k=1}^{n} \sum_{T_{1}, \cdots, T_{k}} \phi^{\left(T_{1}\right)} \cdots \phi^{\left(T_{k}\right)},
$$

where the summation $\sum_{T_{1}, \cdots, T_{k}}$ is over all the partitions $T_{1}, \cdots, T_{k}$ of $\{1, \cdots, n\}$.

For $n=1$ it is trivial to verify (3.7). Suppose that

$$
\frac{\partial^{n-1} Z}{\partial x_{1} \cdots \partial x_{n-1}}=Z \sum_{k=1}^{n-1} \sum_{T_{1}, \cdots, T_{k}} \phi^{\left(T_{1}\right)} \cdots \phi^{\left(T_{k}\right)},
$$

where $T_{1}, \cdots, T_{k}$ is a partition of $\{1, \cdots, n-1\}$. Differentiating (3.8) with respect to $x_{n}$ we get

$$
\begin{aligned}
\frac{\partial^{n} Z}{\partial x_{1} \cdots \partial x_{n-1} \partial x_{n}}= & \sum_{k=1}^{n-1} \sum_{T_{1}, \cdots, T_{k}}\left(\phi^{\left(T_{1}, n\right)} \cdots \phi^{\left(T_{k}\right)}+\cdots+\phi^{\left(T_{1}\right)} \cdots \phi^{\left(T_{k}, n\right)}\right. \\
& \left.+\phi^{\left(T_{1}\right)} \cdots \phi^{\left(T_{k}\right)} \phi^{(n)}\right) Z
\end{aligned}
$$

where $\phi^{\left(T_{h}, n\right)}=\left(\partial \phi^{\left(T_{h}\right)} / \partial x_{n}\right), \phi^{(n)}$ is defined by (3.4) and $T_{h}, n$ is a shorthand notation for $T_{h} \cup\{n\} h=1, \cdots, k$. We observe that in (3.9) the summation over $T_{1}, \cdots, T_{k}$ refers to the partition of $\{1, \cdots, n-1\}$. It is obvious that (3.9) is equal to

$$
Z \sum_{k=1}^{n} \sum_{T_{1}, \cdots, T_{k}} \phi^{\left(T_{1}\right)} \cdots \phi^{\left(T_{k}\right)}
$$

where $\sum_{T_{1} \cdots T_{k}}$ is now over all the partitions $T_{1}, \cdots, T_{k}$ of $\{1, \cdots, n\}$, and (3.7) holds.

ThEOREM 3.1. Let $A=\left(A_{i j}\right)$ be an $n \times n$ matrix over $C$. Then

$$
\operatorname{per}(A)=Z \sum_{k=1}^{n} \sum_{T_{1}, \cdots, T_{k}} \phi^{\left(T_{1}\right)} \cdots \phi^{\left(T_{k}\right)},
$$

where $\phi^{\left(T_{j}\right)}$ is defined by (3.5).

REMARK. The theorem is also true for matrices defined over an arbitrary field if we use formal differentiation.

Now we cast (3.7) into another equivalent form. Then we shall see that $\operatorname{per}(A)$ is in fact independent of $x_{1}, \cdots, x_{n}$. We need the following additional notation. Let $r_{w_{1} \cdots \cdots * w_{s}}$ denote the sum of the entries in the Hadamard product of columns $w_{1}, \cdots, w_{s}$ of $A$, i.e.,

$$
r_{w^{*} \cdots * w_{s}}=\sum_{j=1}^{n} a_{j w_{1}} a_{j w_{2}} \cdots a_{j w_{s}}
$$

Definition 3.1. For $\left(t_{1}, \cdots, t_{k}\right) \in G(n)$ we define the $R$ function, 
$R\left(t_{1}, \cdots, t_{k}\right)$, as the symmetrised sum of all distinct products of the $r_{w_{1} * \cdots * w_{s}}, s=t_{1}, \cdots, t_{k}$, such that in each product the sequences $\left(w_{1}, \cdots, w_{s}\right) \in Q_{s, n}, s=t_{1}, \cdots, t_{k}$, partition the set $\{1, \cdots, n\}$.

Definition 3.2. The permanent of an $m \times n$ matrix $A=\left(a_{i j}\right)$ $m \leqq n$, written $\operatorname{Per}(A)$, is defined by

$$
\operatorname{Per}(A)=\sum_{\sigma} a_{1 \sigma(1)} a_{2 \sigma(2)} \cdots a_{m \sigma(m)},
$$

where the summation extends over all one-to-one functions from $\{1, \cdots, m\}$ into $\{1, \cdots, n\}$.

The special case $m=n$ is of particular importance. In fact most writers restrict the designation "permanent" to the case of square matrices. However the permanent of a rectangular $m \times n$ matrix $A, m \leqq n$, can be obtained by evaluating the permanent of the $n \times n$ matrix

$$
B=\left[\begin{array}{l}
A \\
C
\end{array}\right],
$$

where $C$ is the $(n-m) \times n$ matrix all of whose entries are 1 . Clearly $\operatorname{per}(B)=(n-m) ! \operatorname{per}(A)$. In $[2,1]$ Minc states a formula for the computation of the permanent of an $m \times n$ matrix, $m \leqq n$, in terms of the $R$ functions. This formula is an extension of Binet's formulas for $m \leqq 4$, which are based on the Principle of Inclusion and Exclusion.

If $A$ is an $n \times n$ matrix, then

$$
\operatorname{Per}(A)=\sum_{\left(t_{1}, \cdots, t_{k}\right) \in G(\boldsymbol{x})} C\left(t_{1}, \cdots, t_{k}\right) R\left(t_{1}, \cdots, t_{k}\right)
$$

with

$$
C\left(t_{1}, \cdots, t_{k}\right)=(-1)^{n+k} \prod_{h=1}^{k}\left(t_{h}-1\right) ! \cdot
$$

This is the formula of Binet and Minc of $m=n$. Let us show that (3.7) is precisely this formula.

Let $A^{\prime}=\left(\left(1 / Z_{i}\right) a_{i j}\right)$ be the matrix obtained from $A$ by multiplying its $i$ th row by $1 / Z_{i} i=1, \cdots, n$. Thus, we can write (3.5) in the following form

$$
\phi^{\left(T_{j}\right)}=(-1)^{t_{j}-1}\left(t_{j}-1\right) ! r_{w_{1}^{(j)} * \cdots * w_{s}^{(j)}},
$$

where $r_{w_{1}^{(j)} * \cdots * w_{s}^{(j)}}$ denotes the sum of the entries in the Hadamard product of the columns $w_{1}^{(j)}, \cdots, w_{s}^{(j)}$ of $A, s=t_{j}, j=1, \cdots, k$. On the other hand 


$$
\sum_{k=1}^{n} \sum_{T_{1}, \cdots, T_{k}} \phi^{\left(T_{1}\right)} \cdots \phi^{\left(T_{k}\right)}=\sum_{k=1}^{n} \sum_{\left(t_{1}, \cdots, t_{k}\right)} \sum_{T_{1}, \cdots, T_{k}}^{\prime} \phi^{\left(T_{1}\right)} \cdots \phi^{\left(T_{k}\right)},
$$

where $\Sigma^{\prime}$ denotes the summation over the partitions $T_{1}, \cdots, T_{k}$ whose cardinals $t_{i}=\left|T_{i}\right|, i=1, \cdots, k$, are kept fixed. Substituting (3.10) into (3.11), applying (3.1) and the definition of $R$ we obtain the formula of Binet and Minc for per $\left(A^{\prime}\right)$

$$
\begin{aligned}
& \sum_{k=1}^{n} \sum_{T_{1}, \ldots, T_{k}} \phi^{\left(T_{1}\right)} \cdots \phi^{\left(T_{k}\right)}=\sum_{k=1}^{n} \sum_{T_{1}, \cdots, T_{k}}(-1)^{t_{1}-1} \cdots(-1)^{t_{k}-1}\left(t_{1}-1\right) ! \\
& \times \cdots\left(t_{k}-1\right) ! \prod_{j=1}^{k} r_{w_{1}^{(j)} \cdots * w_{t_{j}}^{(j)}} \\
& =\sum_{k=1}^{n} \sum_{t_{1} \cdots t_{k}}(-1)^{n-k} \prod_{j=1}^{k}\left(t_{j}-1\right) \prod_{j=1}^{k} r_{w_{1}^{(j)} * \cdots * w_{t_{j}}^{(j)}} \\
& =\sum_{\left(t_{1}, \cdots, t_{k}\right) \in G(n)} C\left(t_{1}, \cdots, t_{k}\right) R\left(t_{1}, \cdots, t_{k}\right)=\operatorname{per}\left(A^{\prime}\right) \text {. }
\end{aligned}
$$

It is now clear that in fact (3.7) is independent of $x_{1}, \cdots, x_{n}$ due to the multilinearity of the permanent, since $\operatorname{per}(A)=Z_{1} \cdots Z_{n} \operatorname{per}\left(A^{\prime}\right)=$ $Z \operatorname{per}\left(A^{\prime}\right)$.

4. Ryser's formula. In this section we shall prove Ryser's formula [3] from the identity (2.5), without using the Principle of Inclusion and Exclusion.

THEOREM 4.1. Let $A=\left(a_{i j}\right)$ be a matrix of size $n$ by $n$ defined over a field $F$. Let $w=\left(w_{1}, \cdots, w_{k}\right) \in Q_{k, n}$ and $\bar{w}=\left(\bar{w}_{1}, \cdots, \bar{w}_{n-k}\right) \in$ $Q_{n-k, n}$ be sequences complementary to each other. We shall denote by $A_{w}$ the matrix obtained from $A$ by replacing columns $\bar{w}_{1}, \cdots, \bar{w}_{n-k}$ by zeros. Let $S\left(A_{w}\right)$ represent the product of the row sums of $A_{w}$. Then

$$
\operatorname{per}(A)=\sum_{k=1}^{n} \sum_{w \in Q_{k, n}}(-1)^{n-k} S\left(A_{w}\right)
$$

Proof. Let $A^{\prime}=\left(a_{i j} x_{j}\right)$ be the $n \times n$ matrix obtained from $A$ by multiplying its $j$ th column by $x_{j}, j=1, \cdots, n$. From (2.5) it follows that

$$
\begin{aligned}
S\left(A_{w}^{\prime}\right) & =\prod_{j=1}^{n}\left(\sum_{t=1}^{k} a_{j w_{t}} x_{w_{t}}\right) \\
& =\sum_{\beta} \operatorname{per}\left(A_{w}\left(\beta_{1}, \cdots, \beta_{n}\right)\right) \frac{x_{1}^{\beta_{1}}}{\beta_{1} !} \cdots \frac{x_{n}^{\beta_{n}}}{\beta_{n} !} .
\end{aligned}
$$

Note that if one of the $\beta_{\bar{w}_{t}}, t=1, \cdots, n-k$, is different from zero, the matrix $A_{w}\left(\beta_{1}, \cdots, \beta_{n}\right)$ will have columns of zeros and $\operatorname{per}\left(A_{w}\left(\beta_{1}, \cdots, \beta_{n}\right)\right)=0$. If the condition $\beta_{\bar{w}_{1}}=\cdots=\beta_{\bar{w}_{n-k}}=0$ holds, then $\operatorname{per}\left(A_{w}\left(\beta_{1}, \cdots, \beta_{n}\right)\right)$ will be independent of $w$. 
From (4.2) we have

$$
\begin{aligned}
& \sum_{k=1} \sum_{w \in Q_{k, n}}(-1)^{k} S\left(A_{w}^{\prime}\right) \\
&=\sum_{\beta} \frac{x_{1}^{\beta_{1}}}{\beta_{1} !} \cdots \frac{x_{n}^{\beta_{n}}}{\beta_{n} !} \sum_{k=1}^{n} \sum_{w \in Q_{k}, n}(-1)^{k} \operatorname{per}\left(A_{w}\left(\beta_{1}, \cdots, \beta_{n}\right)\right),
\end{aligned}
$$

where $\sum_{i=1}^{n} \beta_{i}=n$. In order to evaluate (4.3) we first prove that

$$
\sum_{k=1}^{n} \sum_{w \in Q_{k, n}}(-1)^{k} \operatorname{per}\left(A_{w}\left(\beta_{1}, \cdots, \beta_{n}\right)\right)=0
$$

provided that $\beta_{i} \neq 1$ for some $i$. For this purpose let $\left(\beta_{w_{1}}, \cdots, \beta_{w_{h}}\right)$, $w^{\prime}=\left(w_{1}, \cdots, w_{h}\right) \in Q_{h, n}$, are the only nonzero elements in $\left(\beta_{1}, \cdots, \beta_{n}\right)$. It is clear that $\beta_{\bar{w}_{1}}=\cdots=\beta_{\bar{w}_{n-h}}=0$ and $\operatorname{per}\left(A_{w}\left(\beta_{1}, \cdots, \beta_{n}\right)\right)=0$ unless $\bar{w} \subset \bar{w}^{\prime} \Leftrightarrow w \supset w^{\prime}$. Since there are $\left(\begin{array}{l}n-h \\ k-h\end{array}\right)$ different sequences $w, w \in Q_{k, n}$, such that $w \supset w^{\prime}$ for a fixed $w^{\prime}$, we have

$$
\begin{aligned}
\sum_{k=1}^{n} \sum_{w}(-1)^{k} \operatorname{per} & \left(A_{w}\left(\beta_{1}, \cdots, \beta_{n}\right)\right)=\sum_{\substack{k=1 \\
w \supset w^{\prime}}}^{n} \sum_{\substack{w \\
n}}(-1)^{k} \operatorname{per}\left(A\left(\beta_{1}, \cdots, \beta_{n}\right)\right) \\
= & (-1)^{h} \sum_{k=h}^{n}(-1)^{k-h}\left(\begin{array}{l}
n-h \\
k-h
\end{array}\right) \operatorname{per}\left(A\left(\beta_{1}, \cdots, \beta_{n}\right)\right) \\
= & (-1)^{h}(1-1)^{n-h} \operatorname{per}\left(A\left(\beta_{1}, \cdots, \beta_{n}\right)\right) \\
= & 0
\end{aligned}
$$

if $n \neq h$. Consider now the case $n=h$, that is $\beta_{1}=\cdots=\beta_{n}=1$. Clearly $\operatorname{per}\left(A_{w}(1, \cdots, 1)\right)=0$ unless $w=\{1, \cdots, n\}$. From (4.3) and (4.4) it finally follows

$$
\sum_{k=1}^{n} \sum_{w \in Q_{k}, n}(-1)^{k} S\left(A_{w}^{\prime}\right)=(-1)^{n} \operatorname{per}(A) x_{1} \cdots x_{n} .
$$

Since $\operatorname{per}(A)=\left(1 / x_{1} \cdots x_{n}\right)$ per $\left(A^{\prime}\right)$, (4.5) gives Ryser's formula for the evaluation of $\operatorname{per}(A)$.

ACKNOWLEDGMENT. I wish to thank the referee for his careful reading of former versions of this paper. His valuable comments led to a clearer form of the present article.

I am also most greateful to Prof. G. N. de Oliveira for his interest in this work.

\section{REFERENCES}

1. H. Minc, Evaluation of Permanents, Proceedings of the Edinburgh Mathematical Society, 22/1 (1979), 27-32.

2. Encyclopedia of Mathematics and its Applications, vol. 6, Permanents, Addison-Wesley Publishing Company Inc., 1978. 
3. H. Ryser, Combinatorial Mathematics, Carus Mathematical Monographs, John Wiley and Sons, Inc., 1963.

4. J. K. Percus, Combinatorial Methods, Springer Verlag, New.York, 1971.

Received June 22, 1979 and in revised form April 21, 1981.

Universidade de Coimbra

Coimbra, Portugal 



\section{PACIFIC JOURNAL OF MATHEMATICS}

\section{EDITORS}

DONALD BABBITT (Managing Editor)

J. DUGUNDJI

University of California

Los Angeles, California 90024

Hugo Rossi

University of Utah

Salt Lake City, UT 84112

C. C. Moore and Arthur Agus

Department of Mathematics

University of Southern California

Los Angeles, California 90007

R. FinN and J. Milgram

Stanford University

Stanford, California 94305

University of California

Berkeley, CA 94720

\section{ASSOCIATE EDITORS}
R. ARNES
E. F. BeCKenBaCH
B. H. NEUMANN
F. WoLF
K. YoshidA

\section{SUPPORTING INSTITUTIONS}

UNIVERSITY OF ARIZONA

UNIVERSITY OF BRITISH COLUMBIA

CALIFORNIA INSTITUTE OF TECHNOLOGY

UNIVERSITY OF CALIFORNIA

MONTANA STATE UNIVERSITY

UNIVERSITY OF NEVADA, RENO

NEW MEXICO STATE UNIVERSITY

OREGON STATE UNIVERSITY
UNIVERSITY OF OREGON

UNIVERSITY OF SOUTHERN CALIFORNIA

STANFORD UNIVERSITY

UNIVERSITY OF HAWAII

UNIVERSITY OF TOKYO

UNIVERSITY OF UTAH

WASHINGTON STATE UNIVERSITY

UNIVERSITY OF WASHINGTON 


\section{Pacific Journal of Mathematics}

\section{Vol. 101, No. $1 \quad$ November, 1982}

Natália Bebiano, On the evaluation of permanents $\ldots \ldots \ldots \ldots \ldots \ldots \ldots \ldots$

David Borwein and Bruce Brigham Watson, Tauberian theorems between

the logarithmic and Abel-type summability methods $\ldots \ldots \ldots \ldots \ldots \ldots 11$

Leo George Chouinard, II, Hermite semigroup rings $\ldots \ldots \ldots \ldots \ldots \ldots \ldots$

Kun-Jen Chung, Remarks on nonlinear contractions $\ldots \ldots \ldots \ldots \ldots \ldots . \ldots 4$

Lawrence Jay Corwin, Representations of division algebras over local

fields. II ......................................... 49

Mahlon M. Day, Left thick to left lumpy—a guided tour $\ldots \ldots \ldots \ldots \ldots 71$

M. Edelstein and Mo Tak Kiang, On ultimately nonexpansive

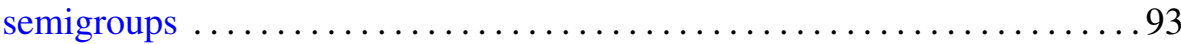

Mary Rodriguez Embry, Semigroups of quasinormal operators . ........ 103

William Goldman and Morris William Hirsch, Polynomial forms on

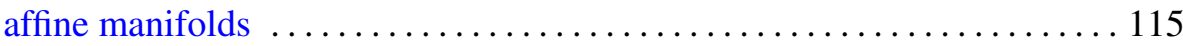

S. Janakiraman and T. Soundararajan, Totally bounded group topologies

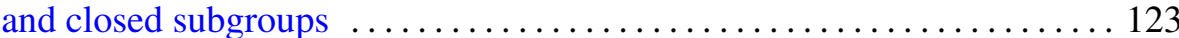

John Rowlay Martin, Lex Gerard Oversteegen and Edward D.

Tymchatyn, Fixed point set of products and cones $\ldots \ldots \ldots \ldots \ldots \ldots 133$

Jan van Mill, A homogeneous Eberlein compact space which is not metrizable ........................................ 141

Steven Paul Plotnick, Embedding homology 3-spheres in $S^{5} \ldots \ldots \ldots \ldots 147$

Norbert Riedel, Classification of the $C^{*}$-algebras associated with minimal rotations

Benedict Seifert, Combinatorial and geometric properties of weight systems of irreducible finite-dimensional representations of simple split Lie algebras over fields of 0 characteristic

James E. Simpson, Dilations on locally convex spaces

Paolo M. Soardi, Schauder bases and fixed points of nonexpansive mappings

Yoshio Tanaka, Point-countable $k$-systems and products of $k$-spaces

Fausto A. Toranzos, The points of local nonconvexity of starshaped sets . . . 209

Lorenzo Traldi, The determinantal ideals of link modules. I . . . . . . . . 215

P. C. Trombi, Invariant harmonic analysis on split rank one groups with applications

Shinji Yamashita, Nonnormal Blaschke quotients 